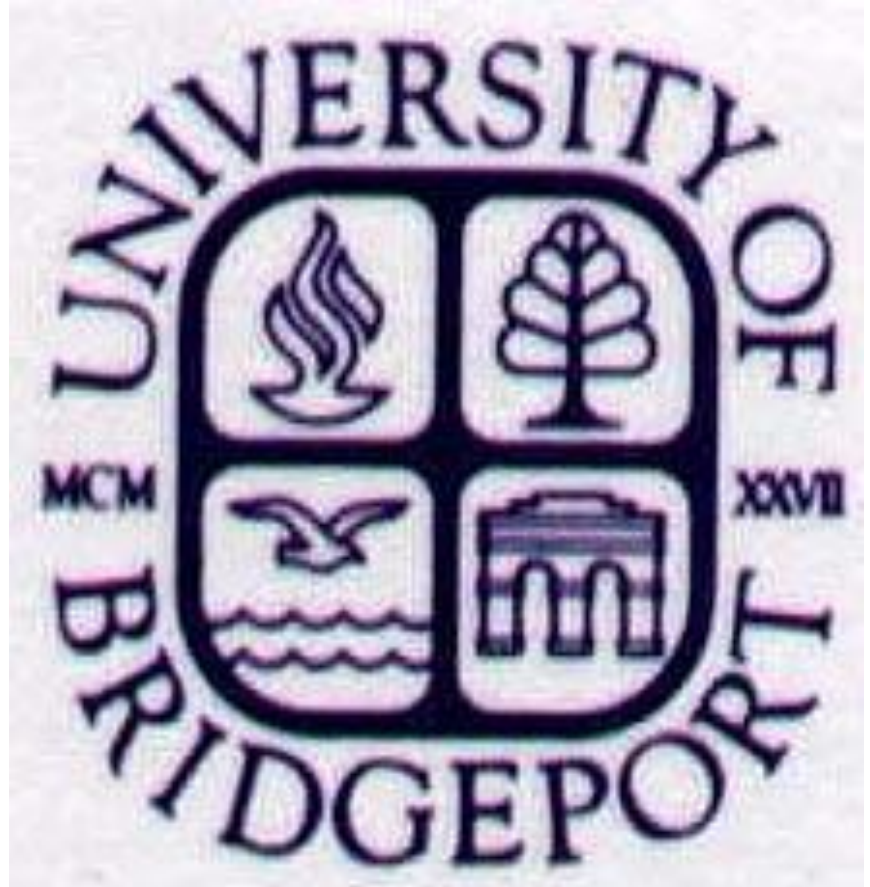

\title{
Histogram Analysis for Automatic Blood Vessels Detection: First Step of IOP
}

Mohammad Al Oudat and Miad Faezipour

Department of Computer Science and Engineering
School of Engineering, University of Bridgeport, CT

\section{Abstract}

Most individuals do not realize they have a broken vein in the eye until somebody lets them know or they look in a mirror. This condition is not tormenting, but commonly creates obtuse trauma to the eye. Treatment is often not required for subconjunctival drainage. In the event that a patient has recognized the presence of blood in his or her eye, it may be fitting for him or her to look for medical consideration. While a subconjunctival drain is occasionally risky, hyphema (blood in the front assembly of the eye, between the cornea and the iris) is conceivably a more serious condition, with more serious outcomes. This work first provides an overview of the most common techniques used to calculate the blood vessels in color images of the retina. Then, it presents a study that has been conducted to discuss the early steps of the intraocular pressure (IOP) detection in the eye, using histogram analysis.

\section{Introduction}

In this advanced time, there are still several ailments that influence diabetes, which in turn, have the tendency to influence various organs of the body like the kidney, heart, and liver. At the point when diabetes influences humans, the veins are generally small in size and henceforth more defenseless. The erosion of veins begins happening when the glucose levels are elevated much over typical levels for an extended period of time. When this happens, the visual fundus image can give data on progressions brought about by various eye diseases, and also provide early indications of certain systemic infections such as diabetes and hypertension. Analyzing fundus pictures has turned into a fundamental and imperative symptomatic system in ophthalmology, and significant efforts have been made to mechanize this procedure. The structure of retinal vessels is a noticeable feature that uncovers additional data on the state of infections, which are reflected as measurable anomalies in distance across shade and tortuosity. Along these lines, dependable routines for vessel recognition that save different vessel estimations are required.

Examination of veins in the eye permits discovery of eye infections such as glaucoma and diabetic retinopathy. Customarily, the vascular system is mapped by hand in a time-devouring process that requires preparation, training and expertise. Computerizing the procedure permits consistency, and in particular, saves the time that a talented specialist would typically use for manual screening. While successful analysis has been attained on ordinary retinal pictures, pictures of anomalous or ailing eyes, for which precision is more pivotal, the calculations oftentimes fall flat. Figure 1 shows the general view of the human eye including the retina, and figure 2 shows the exudate, with the optic disc of the eye organ.
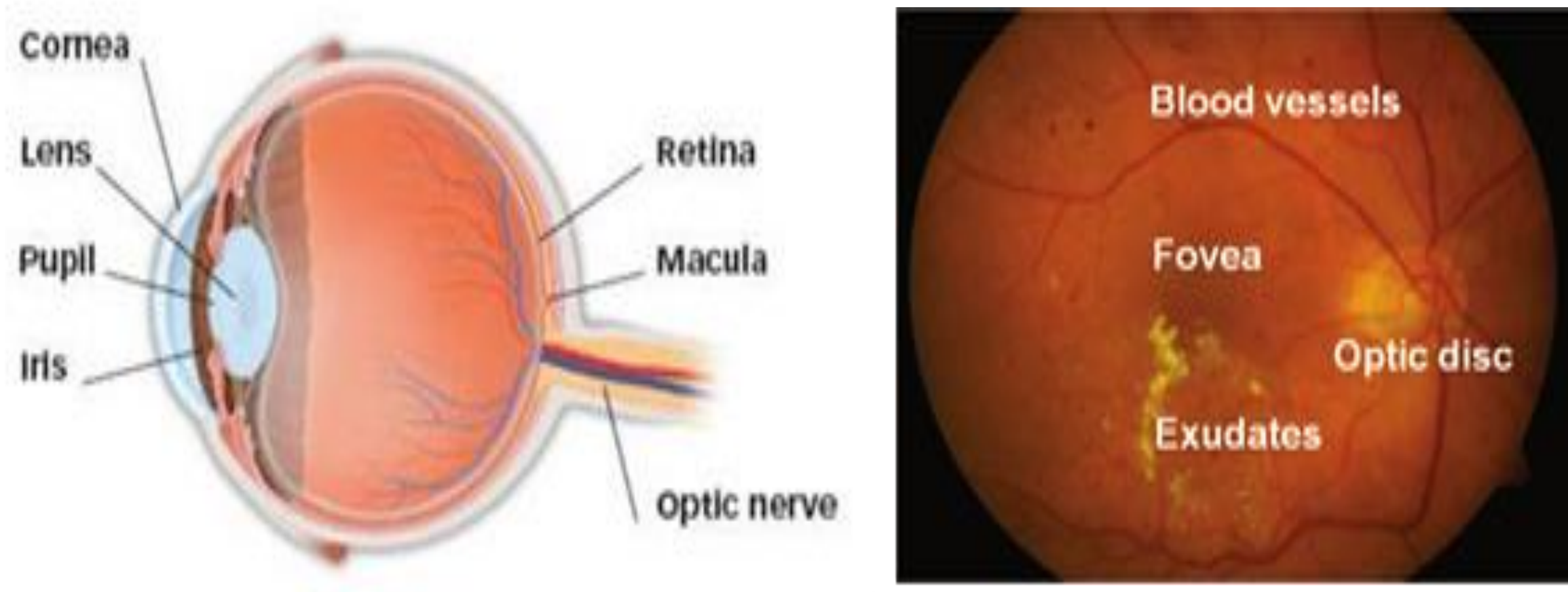

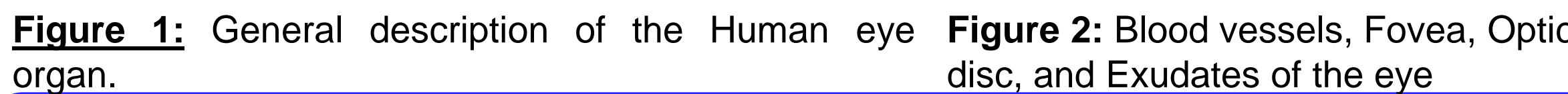
organ.

\section{Proposed Model}

The analysis for automatic blood vessels as the first step in the IOP is detected and checked. Moreover, the simulation was completed by MATLAB 2013a programming. Finally, the process was explained in the next section.

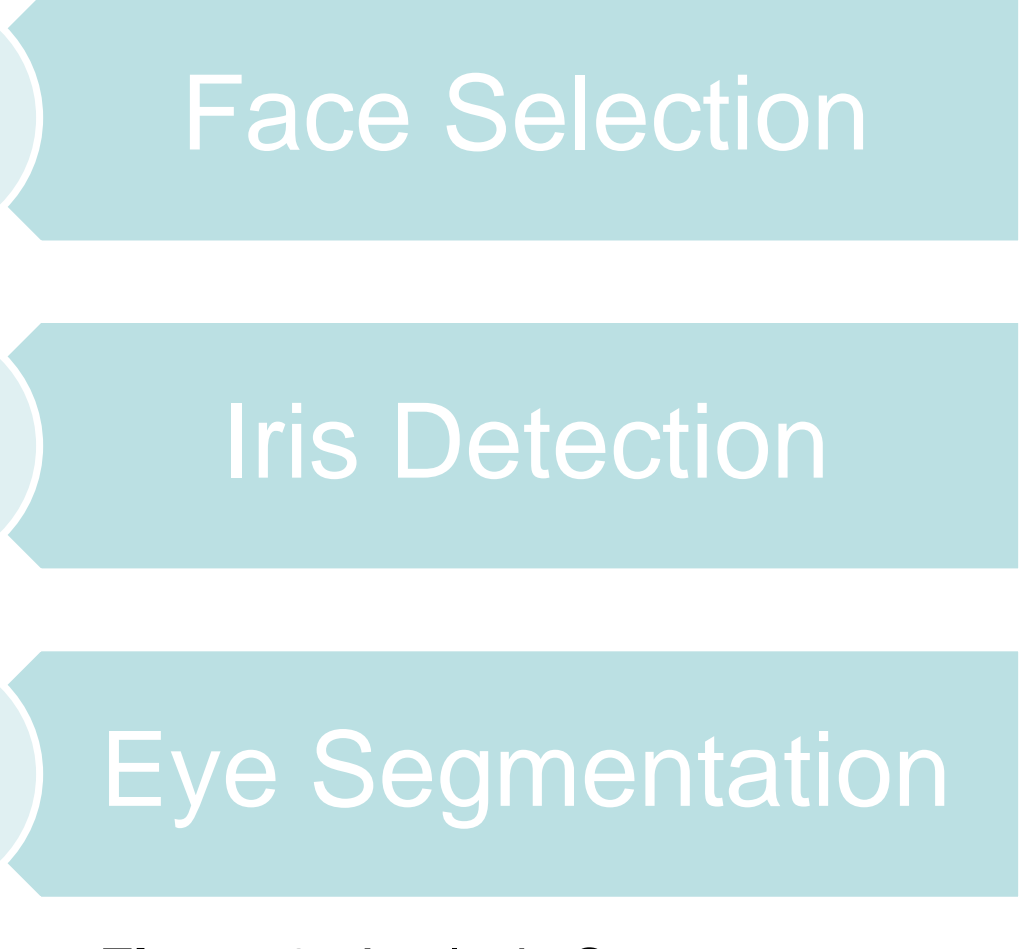

Face Selection

Figure 3: Analysis Steps.

The image database (DB) was taken from patients in a Jordanian Government hospital (Al Ameera Basma Hospital). The patients had ages ranging between 40 and 65 years old, and all of them had eye-related infections or sicknesses. The proposed system is implemented as a real-time face detection and tracking system using 720P(1280×720) @ 30 frames per second (FPS) based on the Viola-Jones face detection algorithm. The color format used to represent the image in each frame is RGB. Figure 3 represents a sample image from the database. All the database images were taken in a range of $20 \mathrm{~cm}$.

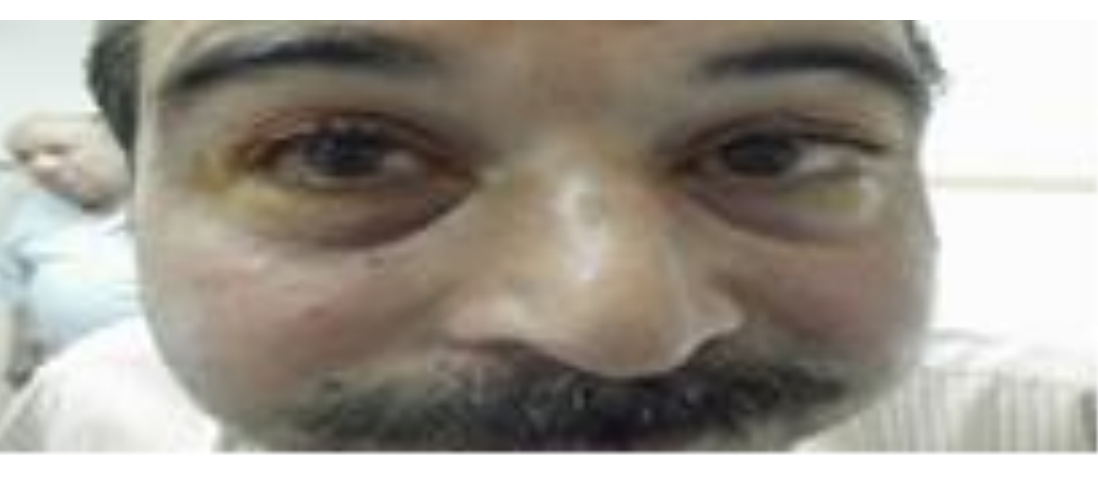

Iris Detection

Figure 4: . Image 1 from the database

The Circular Hough Transform (CHT) has been adopted for iris detection in this system. The Hough transform has been modified into several versions. It has been considered an effective method for detecting curves in images. CHT was presented by A. Herout [16] as a modified version of the original Hough transform. It aims to recognize circular patterns in an image. It is used for transforming sets of feature points residing in the image space into sets of votes that are accumulated in parameter space. Votes are then accumulated for each feature point in an array covering all possible parameter combinations The highest number of votes denotes that a shape is present. The pattern for a circle is defined by Equation 1. Where $x_{0}$ and $y_{0}$ are the center coordinates and $r$ is the radius of the circle.

$$
\left(x_{p}-x_{0}\right)^{2}+\left(y_{p}-y_{0}\right)^{2}=r^{2}
$$
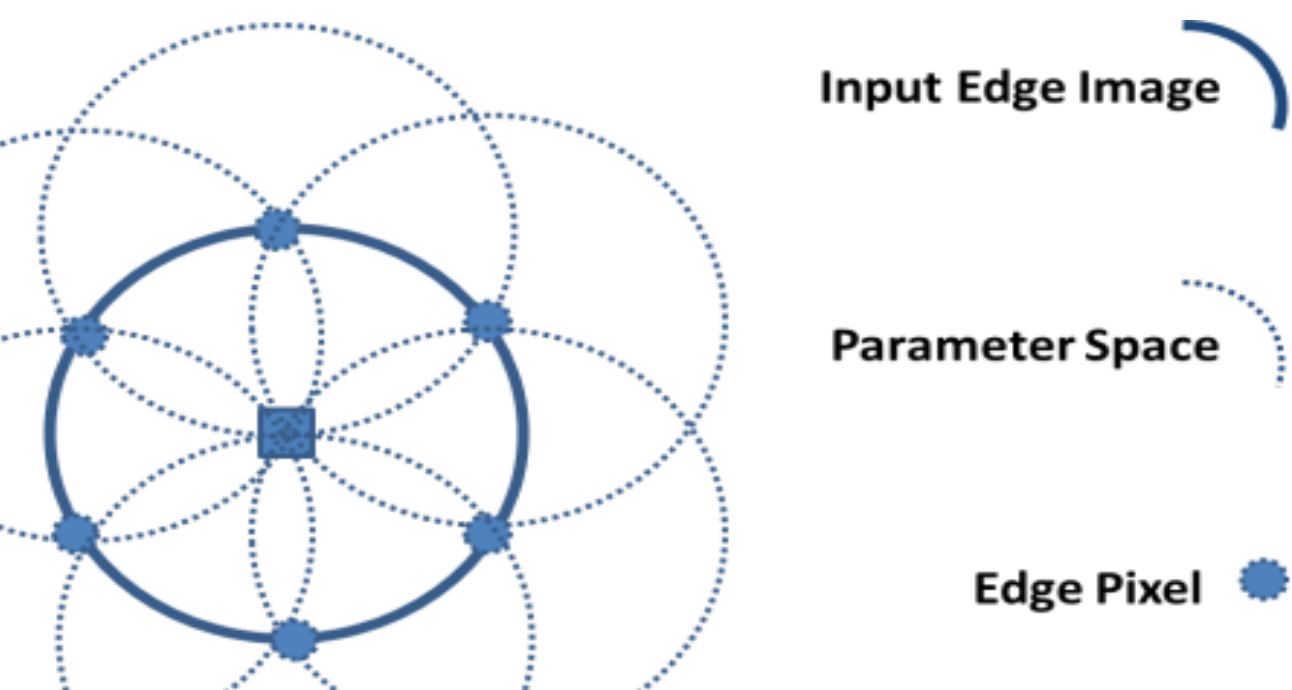

Edge Pixel

\section{Accumulate Point $\square$}

Figure 5: The contribution of the edge points to the accumulator space for $\mathrm{CHT}$.

\section{Eye segmentation}

The presented method is used to segment the blood vessels to overcome the variations in contrast of large and thin vessels. The used method depends on the adaptive thresholding to produce a figure showing the percentage of blood in the retina. The method will then extract large connected components as large vessels. The fragments in the obtained image, including some thin vesse segments (or pixels), are classified by the Support Vector Machine (SVM). The blood vessels tracking process which is the movements of the pupil to all directions to detect the blood vessels is applied to the thin vessel segments to form the whole vascular network.
The use of the histogram in the proposed method allows for probabilities, and is liable to a few conditions. One stipulation is that only nonnegative numbers might be utilized for the scale that provides the tallness of a given bar of the histogram. A second condition is that since likelihood is equivalent to zone, the majority of the ranges of the bars must signify a sum of one equivalent to $100 \%$. The best result quantization map is shown in Figure 6.

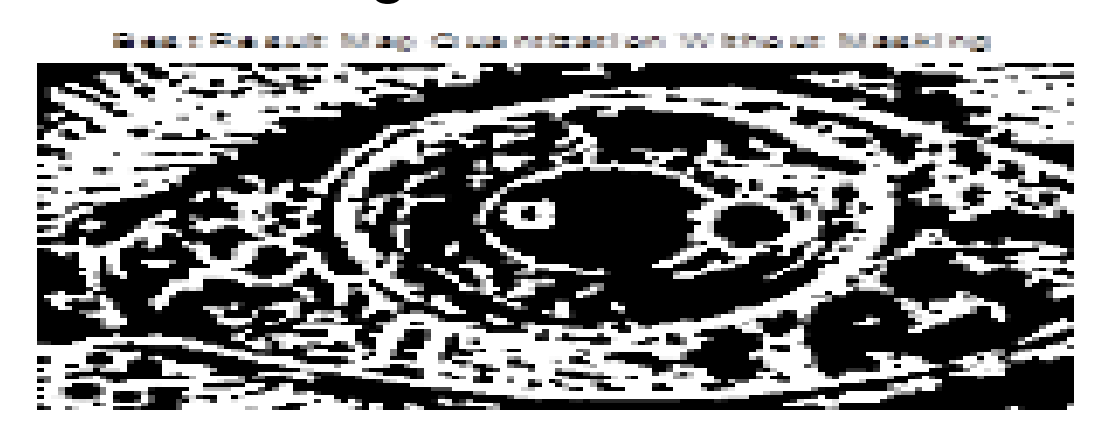

Figure 6. Best Map Quantization without Mask

\section{IOP Cases}

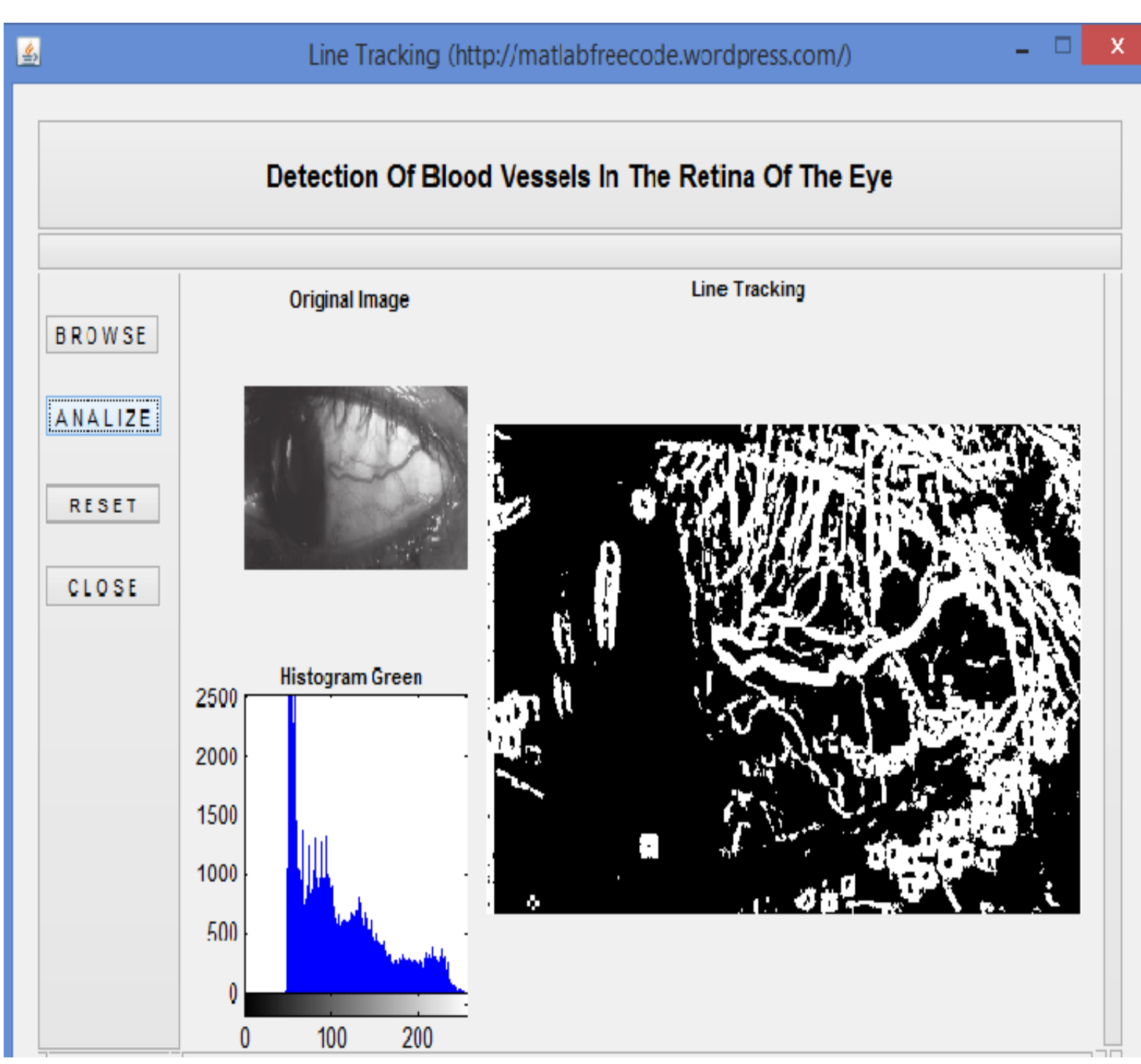

Figure 7. Blood Vessels Detection (IOP)

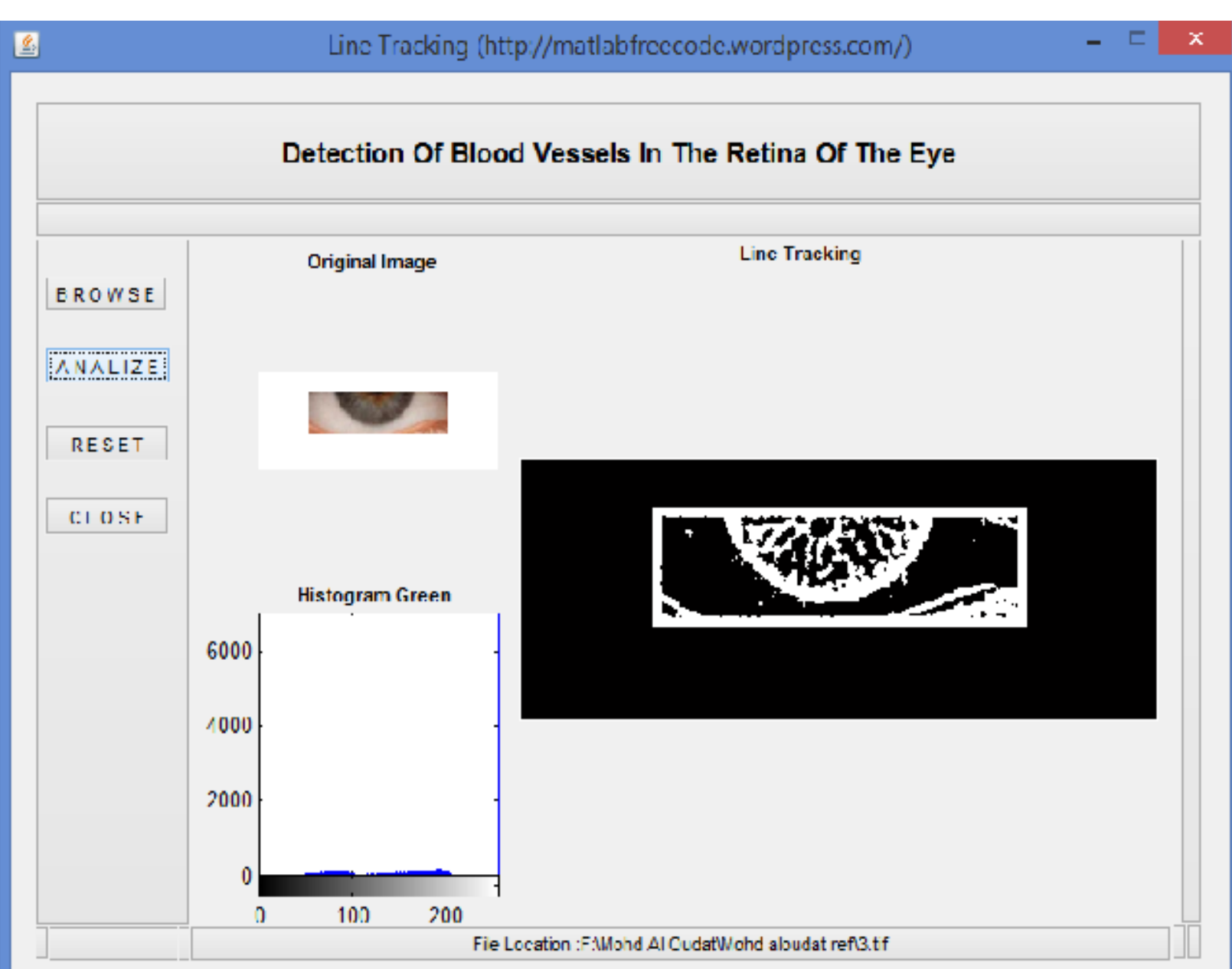

Figure 8. Blood Vessels Detection (No IOP)

\section{Future Work}

An alternate augmentation to the framework would be to compute the rate of the IOP. Presently, as opposed to having a fixed set of iris pictures from a database, an edge grabber could be utilized to catch various pictures, which could lead to enhancing the estimation process. The examination of both eyes to enhance the count system with utilization of a securing cam has the possibility to streamline this operation. For this situation, two formats would be made for every person, one for the left eye and one for the right eye. This design would only acknowledge an individual if both eyes are going to be checked at the same time. The computations delivered for this improvement would need to be adjusted with the expanded imaging if possible because in some cases only one eye has got the higher pressure while the other is normal.

Analyzing two eyes is a good idea, but we should not expect to get the same result, since one eye may have higher pressure than the other. 